\title{
COMPARATIVE MORPHOLOGY OF THE LARYNGEAL MUSCLES IN HYLOBATIDS USING A HIGH-RESOLUTION MRI
}

\author{
TAKESHI NISHIMURA ${ }^{1,{ }^{*}}$, YOSHITAKA NOMURA ${ }^{2}$, HIROHIKO IMAI $^{3}$, TETSUYA $^{2}$ \\ MATSUDA $^{3}$
}

\author{
${ }^{*}$ Corresponding Author: nishimura.takeshi.2r@kyoto-u.ac.jp \\ ${ }^{1}$ Primate Research Institute, Kyoto University, Inuyama, Japan \\ ${ }^{2}$ Graduate School of Science, Kyoto University, Kyoto, Japan \\ ${ }^{3}$ Graduate School of Informatics, Kyoto University, Kyoto, Japan
}

Hylobatids are lesser apes inhabiting the canopy of the tropical forest in Southeast Asia, and they are usually classified into the four independent genera, i.e., Hylobates, Hoolock, Nomascus, Symphalangus. They produce characteristic calls, which are referred to as "song", which comprise a sequence of loud and pure-tone-like voices changing melodiously in fundamental frequency (f0), i.e., pitch. The physiological mechanisms of animal vocalization are often examined by the acoustics of voices recorded in a helium-enriched atmosphere: so-called 'helium voices' (e.g., Nowicki, 1987). The helium voices demonstrated that song voices in gibbons are also produced based on the source-filter independence, like in human speech (Koda et al., 2012). Gibbons use the sound source generated by vibration of the bilateral vocal folds (VFs), to amplify f0 and to suppress the higher harmonics in a filter of the supralaryngeal vocal tract (SVT). This means that modifications of the pitch location principally depend on rapid and flexible modifications of VF elasticity in gibbons. The VF is connected between the anterior commissure of thyroid anteriorly and bilateral arytenoids on the cricoid posteriorly. The VF elasticity is modified by contractions of the vocal muscles that is one of the major components of VF and by varied movements of laryngeal cartilages which extends the VFs in humans. Thus, some anatomical adaptations are expected for large modifications in VF elasticity in gibbons.

We here use the MRI scans of the embalmed specimens of extracted larynges, to examine the morphological variation of laryngeal architecture in gibbons and other non-human primates. The specimens from the four genera of hylobatids, and chimpanzees, orangutans, macaques, were scanned by a high-resolution MRI scanner (7T MRI BioSpec 70/20 USR, circular polarized transceiver volume coil 72-mm bore, T9562), with a special resolution of $54.7-97.7 \mu \mathrm{m}^{3}$ and FOV of 28$50 \mathrm{~mm}^{2}$, depending on specimen's size. Three-dimensional configuration of each 
laryngeal skeleton and internal laryngeal muscle was reconstructed using 3D visualizing software of FEI Amira 6. We also examined the external laryngeal muscles by anatomical dissection.

Some features characteristic and common to hylobatids are found in MRI scans: the vocal muscle is very thin in medio-lateral direction; the first tracheal cartilage is located inside the cricoid cartilage, e.g., intracricoid position; the inner and outer layers of the cricothyroid muscle extend upward and is attached to the middle to superior surface area of thyroid lamina, respectively. By contrast, the other primates have the features that are also found in humans: the thick vocal muscle; the first tracheal cartilage is located inferior to the cricoid; the cricothyroid is attached to the inferior edge or area of the lamina and the inferior horn of thyroid.

The features in gibbons are suggested to underlie their high-pitched and flexible phonation. Their modifications in fo principally depends on the modifications of the spatial relationship of the laryngeal cartilages, e.g., the rocking of the thyroid against the cricoid-arytenoid cartilages rather than of the vocal muscle elasticity. Vocal muscle is very tiny in gibbons, indicating its contraction makes limited contributions to the modifications in the VF elasticity. On the other hand, their long crico-thyroid muscle makes the thyroid rock largely against the cricoid. Such a large rocking requires less efforts for a rapid and repeated modifications in VF elasticity, compared to contractions of the vocal muscle. Such derived features are available for rapid and repeated modifications in pitch in gibbons' song, which is different from mild vocalizations in the other primates examined here.

Humans have anatomical specifications of a two-tube configuration of SVT, which is adapted for rapid and flexible modifications of formants. Gibbons also have another anatomical specification for their melodious singing, but alternatively such specification is not advantageous to mild vocalizations like speech. While humans and gibbons share a common voice physiology, anatomical variation arose adapted for different physiological manipulations of vocalizations varied in primates including humans. Increasing knowledge of the anatomy and physiology in non-human primates promises better understanding of primate origins and of the evolutionary history of physical faculties in human speech.

\section{Acknowledgements}

This study is financially supported in part by JSPS KAKENHI (\#16H04848, TN). We thank Japan Monkey Centre and Yokohama "Zooasia" for a permission to use specimens. 


\section{References}

Koda, H., Nishimura, T., Tokuda, I. T., Oyakawa, C., Nihonmatsu, T., \& Masataka, N. (2012). Soprano singing in gibbons. American Journal of Physical Anthropology, 149, 347-355.

Nowicki, S. (1987). Vocal tract resonances in oscine bird sound production: evidence from birdsongs in a helium atmosphere. Nature, 325, 53-55. 